\title{
Existence and Compactness Results for a System of Fractional Differential Equations
}

\author{
Cheikh Guendouz, ${ }^{1}$ Jamal Eddine Lazreg, ${ }^{1}$ Juan J. Nieto $\mathbb{D}^{2}{ }^{2}$ and Abdelghani Ouahab $\mathbb{D}^{1,3}$ \\ ${ }^{1}$ Laboratory of Mathematics, Sidi-Bel-Abbès University, 89, 22000 Sidi-Bel-Abbès, Algeria \\ ${ }^{2}$ Departamento de Estatística, Análisis Matemático e Optimización, Instituto de Matemáticas, Universidade de Santiago \\ de Compostela, Santiago de Compostela 15782, Spain \\ ${ }^{3}$ Department of Mathematics and Informatics, The African University Ahmed Draia of Adrar, Adrar, Algeria
}

Correspondence should be addressed to Abdelghani Ouahab; agh_ouahab@yahoo.fr

Received 26 January 2020; Accepted 23 May 2020; Published 11 July 2020

Guest Editor: Lishan Liu

Copyright (c) 2020 Cheikh Guendouz et al. This is an open access article distributed under the Creative Commons Attribution License, which permits unrestricted use, distribution, and reproduction in any medium, provided the original work is properly cited.

\begin{abstract}
The existence and uniqueness, boundedness, and continuous dependence of solutions for fractional differential equations with Caputo fractional derivative is proven by Perov's fixed point theorem in vector Banach spaces. We study the existence and compactness of solution sets and the u.s.c. of operator solutions.
\end{abstract}

\section{Introduction}

In the past twenty years, the fractional differential equation has aroused great consideration not only in its application in mathematics but also in other applications in physics, engineering, finance, fluid mechanics, viscoelastic mechanics, electroanalytical chemistry, and biological and other sciences [1-7].

In recent decades, the Riemann-Liouville, Caputo, and Hadamard fractional calculus are paid more attention; see the monographs [5, 8-13].

Applied problems requiring definitions of fractional derivatives are those that are physically interpretable for initial conditions containing $y(0), y^{\prime}(0)$, etc. The same requirements are true for boundary conditions. Caputo's fractional derivative satisfies these demands. For more details on the geometric and physical interpretation for fractional derivatives of both the Riemann-Liouville type and the Caputo type, see Podlubny [12] and Diethelm [14].

The theory of fractional differential equations and inclusions has been extensively studied and developed by many authors; see [15-21] and the references therein.
Perov in 1964 [22] and Perov and Kibenko [23] extended the classical Banach contraction principle for contractive maps on space endowed with a vector-valued metric. Later, they attempted to generalize the Perov fixed point theorem in several directions which has a number of applications in various fields of nonlinear analysis, semilinear differential equations, and system of ordinary differential equations.

In [24], Dezideriu and Precup studied the following system of semilinear equations

$$
\left\{\begin{array}{l}
A_{1} x=F^{1}(x, y) \\
A_{2} y=F^{2}(x, y)
\end{array}\right.
$$

where $A_{1}, A_{2}: D(A) \subset X \longrightarrow X$ are linear operators and $F^{1}$, $F^{2}: J \times X \times X \longrightarrow X$ are nonlinear operators.

Precup, in [25], explained the advantage of vector-valued norms and the role of matrices that are convergent to zero in the study of semilinear operator systems.

Many authors studied the existence of solutions for a system of differential equations and impulsive differential 
equations by using the vector version fixed point theorem; their results are given in [26-30].

Our goal of this paper is to treat the systems of fractional differential equations. More precisely, we will consider the following problem:

$$
\left\{\begin{array}{l}
{ }^{c} D^{\alpha} x(t)=f(t, x(t), y(t)), \\
{ }^{c} D^{\beta} y(t)=g(t, x(t), y(t)), \quad t \in J, \\
x(0)=x_{0}, \\
y(0)=y_{0},
\end{array}\right.
$$

where ${ }^{c} D^{\alpha}$ and ${ }^{c} D^{\beta}$ are the Caputo fractional derivatives, $\alpha$, $\beta \in(0,1], J=[0, \infty), f, g: J \times \mathbb{R} \times \mathbb{R} \longrightarrow \mathbb{R}$ are given functions, and $x_{0}, y_{0} \in \mathbb{R}$.

In the case where $\alpha=\beta=1$, the above system was used to analyze initial value problems and boundary value problems for nonlinear competitive or cooperative differential systems from mathematical biology [31] and mathematical economics [32] which can be set in the operator from $((2))$.

The plan of this paper is as follows: in Section 2, we introduce all the background material used in this paper such as some properties of generalized Banach spaces, fixed point theory, and fractional calculus theory. In Section 3, we state and prove our main results by using Perov's fixed point type theorem in generalized Banach spaces. By the Leray-Schauder fixed point in vector Banach space, we prove the existence and compactness of solution sets of the above problems.

\section{Preliminaries}

In this section, we introduce notations, definitions, and preliminary facts which are used throughout this paper.

Definition 1 [22]. Let $X$ be a nonempty set. The mapping $d: X \times X \longrightarrow \mathbb{R}_{+}^{m}$ which satisfies all the usual axioms of the metric is called a generalized metric in Perov's sense and $(X, d)$ is called a generalized metric space.

In a generalized metric space in Perov's sense, the concepts of Cauchy sequence, convergent sequence, completeness, and open and closed subsets are similarly defined as those for usual metric space.

If $v, r \in \mathbb{R}^{m}, v:=\left(v_{1}, v_{2}, \cdots, v_{m}\right)$ and $r:=\left(r_{1}, r_{2}, \cdots, r_{m}\right)$, then by $v \leq r$, we mean $v_{i} \leq r_{i}$ for each $i \in\{1, \cdots, m\}$, and by $v<r$, we mean $v_{i}<r_{i}$ for each $i \in\{1, \cdots, m\}$. Also $|v|:=$ $\left(\left|v_{1}\right|, \cdots,\left|v_{m}\right|\right)$ and $\max (u, v):=\left(\max \left(u_{1}, v_{1}\right), \cdots, \max \left(u_{m}\right.\right.$, $\left.v_{m}\right)$ ). If $c \in \mathbb{R}$, then $v \leq c$ means $v_{i} \leq c$ for each $i \in\{1, \cdots, m\}$. Denote by

$$
B\left(x_{0}, r\right)=\left\{x \in X: d\left(x_{0}, x\right)<r\right\},
$$

the open ball centered in $x_{0}$ with radius $r$, and

$$
\overline{B\left(x_{0}, r\right)}=\left\{x \in X: d\left(x_{0}, x\right) \leq r\right\}
$$

the closed ball centered in $x_{0}$ with radius $r$.
Definition 2. A square matrix $M$ of real numbers is said to be convergent to zero if and only if $M^{n} \longrightarrow 0$ as $n \longrightarrow \infty$.

Lemma 3 [33]. Let $M \in \mathscr{M}_{m, m}\left(\mathbb{R}_{+}\right)$. The following statements are equivalent:

(i) $M$ is a matrix convergent to zero

(ii) The eigenvalues of $M$ are in open disc, i.e., $|\mu|<1$, for every $\mu \in \mathbb{C}$ with $\operatorname{det}(M-\mu I)=0$

(iii) The matrix $I-M$ is nonsingular and $(I-M)^{-1}=$ $I+M+\cdots+M^{n}+\cdots$

(iv) The matrix $I-M$ is nonsingular and $(I-M)^{-1}$ has nonnegative elements

(v) $M^{n} q \longrightarrow 0$ and ${ }^{t} q M^{n} \longrightarrow 0$ as $n \longrightarrow \infty$, for any $q \in \mathbb{R}^{m}$

Example 4. Some examples of matrix convergent to zero are

$$
\text { 1) } M=\left(\begin{array}{ll}
a & b \\
a & b
\end{array}\right) \text {, }
$$

where $a, b \in \mathbb{R}_{+}$and $a+b<1$,

$$
\text { 2) } M=\left(\begin{array}{ll}
a & a \\
b & b
\end{array}\right) \text {, }
$$

where $a, b \in \mathbb{R}_{+}$and $a+b<1$, and

$$
\text { 3) } M=\left(\begin{array}{ll}
a & b \\
0 & c
\end{array}\right) \text {, }
$$

where $a, b, c \in \mathbb{R}_{+}$and $\max \{a, c\}<1$.

Definition 5 [34]. Let $(X, d)$ be a generalized metric space. An operator $N: X \longrightarrow X$ is said to be contractive if there exists a convergent to zero matrix $M$ such that

$$
d(N(x), N(y)) \leq M d(x, y) \quad \text { for all } x, y \in X .
$$

Notice now that the Banach fixed point theorem can be extended to generalized metric spaces in the sense of Perov.

Theorem $6[22,28]$. Let $(X, d)$ be a complete generalized metric space and $N: X \longrightarrow X$ be a contractive operator with Lipschitz matrix $M$. Then, $N$ has a unique fixed point $x^{*}$, and for each $x_{0} \in X$, we have

$$
\left.d\left(N^{k}\left(x_{0}\right), x^{*}\right)\right) \leq M^{k}(I-M)^{-1} d\left(x_{0}, N\left(x_{0}\right)\right) \quad \text { for all } k \in \mathbb{N} \text {. }
$$

We recall now the following Leary-Schauder type theorem. 
Theorem $7[28,35]$. Let $X$ be a generalized Banach space and let $N: X \longrightarrow X$ be a completely continuous operator. Then, either

(i) the equation $N(x)=x$ has at least one solution, or

(ii) the set $\mathscr{M}=\{x \in X \mid \mu N(x)=x, \mu \in(0,1)\} \quad$ is unbounded

We will use the following notations. Let $(X, d)$ and $(Y, \rho)$ be two metric spaces and $G: X \longrightarrow \mathscr{P}(Y)$.

$$
\begin{aligned}
\mathscr{P}(X) & =\{Y \subset X: Y=\varnothing\}, \mathscr{P}_{c l}(X) \\
& =\{Y \in \mathscr{P}(X): Y \text { is closed }\}, \mathscr{P}_{b}(X) \\
& =\{Y \in \mathscr{P}(X): Y \text { bounded }\} .
\end{aligned}
$$

Definition $8[28,36]$. A multivalued map $\varphi: X \longrightarrow \mathscr{P}(Y)$ is called upper semicontinuous (u.s.c.) at a point $x_{0} \in X$ provided that for every open subset $V \subset Y$ with $V \supseteq \varphi\left(x_{0}\right)$, there exists $U \in \mathscr{V}\left(x_{0}\right)$ such that

$$
\forall x \in U, \varphi(x) \subset V
$$

$\varphi$ is called upper semicontinuous if it is u.s.c. at every point $x \in X$.

The mapping $G$ is said to be completely continuous if it is u.s.c., and for every bounded subset $C \subseteq X, G(C)$ is relatively compact, i.e., there exists a relatively compact set $K=K \subset X$ such that

$$
G(C)=\cup\{G(x): x \in C\} \subset K \text {. }
$$

Also, $G$ is compact if $G(X)$ is relatively compact, and it is called locally compact if for each $x \in X$, there exists an open set $W$ containing $x$, such that $G(W)$ is relatively compact.

Theorem 9 [36]. Let $G: X \longrightarrow \mathscr{P}_{c p}(Y)$ be a closed locally compact multifunction. Then, $G$ is u.s.c.

Now, we recall some notations and definitions of fractional calculus theory.

Definition 10 [5]. The Riemann-Liouville fractional integral of the function $h \in L^{1}\left([0, T], \mathbb{R}_{+}\right)$of order $\alpha \in \mathbb{R}_{+}$is defined by

$$
I^{a} h(t)=\frac{1}{\Gamma(a)} \int_{0}^{t}(t-s)^{a-1} h(s) d s
$$

where $\Gamma$ is the Euler gamma function defined by $\Gamma(a)=$ $\int_{0}^{\infty} t^{\alpha-1} e^{-t} d t, \alpha>0$.
Definition 11 [5]. For a function $h \in A C^{n}(J, \mathbb{R})$, the Caputo fractional-order derivative of order $\alpha$ of $h$ is defined by

$$
\left({ }^{c} D_{0}^{\alpha} h\right)(t)=\frac{1}{\Gamma(n-\alpha)} \int_{0}^{t}(t-s)^{n-\alpha-1} h^{(n)}(s) d s,
$$

where $n=[\alpha]+1$.

We recall Gronwall's lemma for singular kernels, whose proof can be found in Lemma 7.1.1 of [37].

Lemma 12. Let $v:[0, b) \longrightarrow[0, \infty)$ be a real function; $w(\cdot)$ is a nonnegative, locally integrable function on $[0, b)$ (some $b \leq$ $+\infty) ; a(t)$ is a nonnegative, nondecreasing continuous function defined on $0 \leq t<b ; a(t) \leq M$ (constant); and suppose $v$ $(t)$ is nonnegative and locally integrable on $0 \leq t<b$. Assume $\gamma>0$ such that

$$
v(t) \leq w(t)+a(t) \int_{0}^{t} \frac{v(s)}{(t-s)^{1-\gamma}} d s,
$$

then

$$
v(t) \leq w(t)+\int_{0}^{t} \sum_{n=1}^{\infty} \frac{(a(t) \Gamma(\gamma))^{n}}{\Gamma(n \gamma)}(t-s)^{n \gamma-1} w(s) d s,
$$

for every $t \in[0, b)$

\section{Existence, Uniqueness, and Bounded Solutions}

In order to define a solution for problem (2), consider the following functional spaces. Let $J=[0, \infty)$ and $C(J, \mathbb{R})$ be the space of all continuous functions from $J$ into $\mathbb{R}$.

$$
C_{b}=\{y \in C(J, \mathbb{R}): y \text { is bounded }\} \text {. }
$$

$C_{b}$ is a Banach with norm

$$
\|y\| C_{b}=\sup \{|y(t)|: t \in J\}
$$

We need the following auxiliary result.

Lemma 13 [14]. Concerning the problem,

$$
\left\{\begin{array}{l}
{ }^{c} D^{\alpha} x(t)=f(t, x), \quad 0<\alpha<1, \\
x(0)=x_{0} \in \mathbb{R},
\end{array}\right.
$$

where the function $f: \mathbb{R}_{+} \times \mathbb{R} \longrightarrow \mathbb{R}$ is continuous. The function $x: \mathbb{R}_{+} \times \mathbb{R} \longrightarrow \mathbb{R}$ is the unique solution of the problem (19) if and only if

$$
x(t)=x_{0}+\frac{1}{\Gamma(\alpha)} \int_{0}^{t}(t-s)^{\alpha-1} f(s, x(s)) d s, \quad t \in \mathbb{R}_{+} .
$$


Definition 14. A function $x, y \in C_{b}$ is said to be a solution of (2) if and only if

$$
\left\{\begin{array}{l}
x(t)=x_{0}+\frac{1}{\Gamma(\alpha)} \int_{0}^{t}(t-s)^{\alpha-1} f(s, x(s), y(s)) d s, \\
y(t)=y_{0}+\frac{1}{\Gamma(\beta)} \int_{0}^{t}(t-s)^{\beta-1} g(s, x(s), y(s)) d s, \\
t \in J .
\end{array}\right.
$$

In this section, we assume the following conditions.

(H1). There exists functions $h_{i, \alpha}, h_{j, \beta} \in L^{1}\left(J, \mathbb{R}^{+}\right), \quad i=1,2$, $j=3,4$ such that

$$
\begin{aligned}
& |f(s, x, y)-f(s, \bar{x}, \bar{y})| \\
& \quad \leq h_{i, \alpha}(s)|x-\bar{x}|+h_{2, \alpha}(s)|y-\bar{y}| \quad \text { for all } x, \bar{x}, y, \bar{y} \in \mathbb{R}, \\
& |g(s, x, y)-g(s, \bar{x}, \bar{y})| \\
& \quad \leq h_{3, \beta}(s)|x-\bar{x}|+h_{4, \beta}(s)|y-\bar{y}| \quad \text { for all } x, \bar{x}, y, \bar{y} \in \mathbb{R},
\end{aligned}
$$

where

$$
\begin{aligned}
& \left\|h_{i, \alpha}\right\|_{\infty}=\sup _{t \in J} \int_{0}^{t} \theta^{\alpha}(t-s) h_{i, \alpha}(s) d s<\infty, \quad i=1,2, \\
& \left\|h_{j, \beta}\right\|_{\infty}=\sup _{t \in J} \int_{0}^{t} \theta^{\beta}(t-s) h_{j, \beta}(s) d s<\infty, \quad j=3,4,
\end{aligned}
$$

where for $\gamma=\alpha, \beta$,

$$
\theta_{\gamma}(t)= \begin{cases}t^{\gamma-1}, & t>0 \\ 0, & t \leq 0\end{cases}
$$

(H2). The functions $f_{\alpha}, g_{\beta}: J \longrightarrow \mathbb{R}$ are defined by

$$
\begin{aligned}
f_{\alpha}(t) & =\theta_{\alpha}(t-s) f(s, 0,0), g_{\beta}(t) \\
& =\theta_{\beta}(t-s) g(s, 0,0), \quad t \in J,
\end{aligned}
$$

satisfies

$$
\begin{aligned}
& \left\|f_{\alpha}\right\|_{\infty}=\sup _{t \in J} \int_{0}^{t} \theta_{\alpha}(t-s)|f(s, 0,0)| d s<\infty, \\
& \left\|g_{\beta}\right\|_{\infty}=\sup _{t \in J} \int_{0}^{t} \theta_{\beta}(t-s)|g(s, 0,0)| d s<\infty .
\end{aligned}
$$

Now, we are in a position to prove our existence and uniqueness solution for the problem (2) using the Perov fixed point theorem and show that for each initial condition $\left(x_{0}\right.$, $\left.y_{0}\right)$, the solution is bounded.
Theorem 15. Assume that (H1)-(H2) are satisfied. If the matrix

$$
M=\left(\begin{array}{cc}
\frac{\left\|h_{1, \alpha}\right\|_{\infty}}{\Gamma(\alpha)} & \frac{\left\|h_{2, \alpha}\right\|_{\infty}}{\Gamma(\alpha)} \\
\frac{\left\|h_{3, \beta}\right\|_{\infty}}{\Gamma(\beta)} & \frac{\left\|h_{4, \beta}\right\|_{\infty}}{\Gamma(\beta)}
\end{array}\right) \in \mathscr{M}_{2 \times 2}\left(\mathbb{R}^{+}\right),
$$

converges to zero. Then, the problem (2) has a unique bounded solution.

Proof. Transform the problem (2) into a fixed point theorem of the operator $N: C_{b} \times C_{b} \longrightarrow C_{b} \times C_{b}$ defined by $N(x, y)$ $=\left(N_{1}(x, y), N_{2}(x, y)\right)$, where

$$
\begin{gathered}
N_{1}(x, y)(t)=x_{0}+\frac{1}{\Gamma(\alpha)} \int_{0}^{t}(t-s)^{\alpha-1} f(s, x(s), y(s)) d s \\
N_{2}(x, y)(t)=y_{0}+\frac{1}{\Gamma(\beta)} \int_{0}^{t}(t-s)^{\beta-1} g(s, x(s), y(s)) d s \\
t \in[0, \infty) .
\end{gathered}
$$

First, we show that the operator $N$ is well-defined. Let $(x, y) \in C_{b} \times C_{b}$ and $t \in[0, \infty)$, then we have

$$
\begin{aligned}
\left|N_{1}(x, y)(t)\right| \leq & \left|x_{0}\right|+\frac{1}{\Gamma(\alpha)} \int_{0}^{t}(t-s)^{\alpha-1}|f(s, x(s), y(s))| d s \\
\leq & \left|x_{0}\right|+\frac{1}{\Gamma(\alpha)} \int_{0}^{t}(t-s)^{\alpha-1} \\
& \cdot|f(s, x(s), y(s))-f(s, 0,0)| d s \\
& +\frac{1}{\Gamma(\alpha)} \int_{0}^{t} \frac{|f(s, 0,0)|}{(t-s)^{1-\alpha}} d s .
\end{aligned}
$$

Then,

$$
\begin{array}{r}
\left\|N_{1}(x, y)\right\|_{c_{b}} \leq\left|x_{0}\right|+\frac{\left\|h_{1, \alpha}\right\|_{\infty}}{\Gamma(\alpha)}\|x\| c_{b} \\
+\frac{\left\|h_{2, \alpha}\right\|_{\infty}}{\Gamma(\alpha)}\|y\| c_{b}+\frac{\left\|f_{\alpha}\right\|_{\infty}}{\Gamma(\alpha)}, \\
\left\|N_{2}(x, y)\right\|_{c_{b}} \leq\left|y_{0}\right|+\frac{\left\|h_{3, \beta}\right\|_{\infty}}{\beta}\|x\| c_{b} \\
+\frac{\left\|h_{4, \beta}\right\|_{\infty}}{\beta}\|y\| c_{b}+\frac{\left\|g_{\beta}\right\|_{\infty}}{\Gamma(\beta)} .
\end{array}
$$

Hence, the operator $N$ is well-defined.

Clearly, the fixed points of operator $N$ are solutions of problem (2). Now, we show that $N$ is a contraction. For all $(x, y),(\bar{x}, \bar{y}) \in C_{b} \times C_{b}$, we have 


$$
\begin{aligned}
& \left|N_{1}(x, y)(t)-N_{1}(\bar{x}, \bar{y})(t)\right| \\
& \quad \leq \frac{1}{\Gamma(\alpha)} \int_{0}^{t}(t-s)^{\alpha-1}|f(s, x(t), y(t))-f(s, \bar{x}(t), \bar{y}(t))| d s \\
& \quad \leq \frac{1}{\Gamma(\alpha)} \int_{0}^{t}\left[h_{1, \alpha}(s)|x(t)-\bar{x}(t)|+h_{2, \alpha}(s)|y(t)-\bar{y}(t)|\right] d s .
\end{aligned}
$$

Then,

$$
\begin{aligned}
& \left\|N_{1}(x, y)-N_{1}(\bar{x}, \bar{y})\right\| c_{b} \\
& \quad \leq \frac{\left\|h_{1, \alpha}\right\|_{\infty}}{\Gamma(\alpha)}\|x-\bar{x}\| c_{b}+\frac{\left\|h_{2, \alpha}\right\|_{\infty}}{\Gamma(\alpha)}\|y-\bar{y}\| c_{b} .
\end{aligned}
$$

Similarly, we have

$$
\begin{aligned}
& \left\|N_{2}(x, y)-N_{2}(\bar{x}, \bar{y})\right\|_{C_{b}} \\
& \quad \leq \frac{\left\|h_{3, \beta}\right\|_{\infty}}{\Gamma(\beta)}\|x-\bar{x}\|_{C_{b}}+\frac{\left\|h_{4, \beta}\right\|_{\infty}}{\Gamma(\beta)}\|y-\bar{y}\|_{C_{b}} .
\end{aligned}
$$

Therefore,

$$
\|N(x, y)-N(\bar{x}, \bar{y})\|_{C_{b} \times C_{b}} \leq M\left(\begin{array}{c}
\|x-\bar{x}\|_{C_{b}} \\
\|y-\bar{y}\|_{C_{b}}
\end{array}\right) .
$$

According to Theorem 6, we deduce that the operator $N$ has unique fixed point which is a solution of problem (2). Now, we will prove that the solution $(x, y)$ of problem (2) is bounded. For all $t \in[0, \infty)$, we have

$$
\begin{aligned}
&|x(t)| \leq\left|x_{0}\right|+\frac{1}{\Gamma(\alpha)} \int_{0}^{t}(t-s)^{\alpha-1}|f(s, x(s), y(s))| d s \\
& \leq\left|x_{0}\right|+\frac{1}{\Gamma(\alpha)} \int_{0}^{t}\left[\left(h_{1, \alpha}(s)|x(s)|+h_{2, \alpha}(s)|y(s)|\right] d s\right. \\
&+\frac{1}{\Gamma(\alpha)} \int_{0}^{t}\left|f_{\alpha}(s, 0,0)\right| d s \\
& \leq\left|x_{0}\right|+\frac{1}{\Gamma(\alpha)} \int_{0}^{t}\left[\left(h_{1, \alpha}(s)|x(s)|+h_{2, \alpha}(s)|y(s)|\right] d s+\frac{\left\|f_{\alpha}\right\|_{\infty}}{\Gamma(\alpha)},\right. \\
&|y(t)| \leq\left|y_{0}\right|+\frac{1}{\Gamma(\beta)} \int_{0}^{t}(t-s)^{\beta-1}|g(s, x(s), y(s))| d s \\
& \leq\left|y_{0}\right|+\frac{1}{\Gamma(\beta)} \int_{0}^{t}\left[\left(h_{3, \beta}(s)|x(s)|+h_{4, \beta}(s)|y(s)|\right] d s\right. \\
& \quad+\frac{1}{\Gamma(\beta)} \int_{0}^{t}\left|g_{\beta}(s, 0,0)\right| d s \\
& \leq\left|y_{0}\right|+\frac{1}{\Gamma(\beta)} \int_{0}^{t}\left[\left(h_{3, \beta}(s)|x(s)|+h_{4, \alpha}(s)|y(s)|\right] d s+\frac{\left\|g_{\beta}\right\|_{\infty}}{\Gamma(\beta)} .\right.
\end{aligned}
$$

Therefore,

$$
\begin{aligned}
& \|x\|_{C_{b}} \leq\left|x_{0}\right|+\frac{\left\|h_{1, \alpha}\right\|_{\infty}}{\Gamma(\alpha)}\|x\|_{C_{b}}+\frac{\left\|h_{2, \alpha}\right\|_{\infty}}{\Gamma(\alpha)}\|y\|_{C_{b}}+\frac{\left\|f_{\alpha}\right\|_{\infty}}{\Gamma(\alpha)}, \\
& \|y\|_{C_{b}} \leq\left|y_{0}\right|+\frac{\left\|h_{3, \beta}\right\|_{\infty}}{\Gamma(\beta)}\|x\|_{C_{b}}+\frac{\left\|h_{4, \beta}\right\|_{\infty}}{\Gamma(\beta)}\|y\|_{C_{b}}+\frac{\left\|g_{\beta}\right\|_{\infty}}{\Gamma(\beta)} .
\end{aligned}
$$

Hence,

$$
\left(\begin{array}{c}
\|x\|_{C_{b}} \\
\|y\|_{C_{b}}
\end{array}\right) \leq M\left(\begin{array}{l}
\|x\|_{C_{b}} \\
\|y\|_{C_{b}}
\end{array}\right)+\left(\begin{array}{c}
\sigma_{1} \\
\sigma_{2}
\end{array}\right)
$$

where

$$
\sigma_{1}=\left|x_{0}\right|+\frac{\left\|f_{\alpha}\right\|_{\infty}}{\Gamma(\alpha)}, \sigma_{2}=\left|y_{0}\right|+\frac{\left\|g_{\beta}\right\|_{\infty}}{\Gamma(\beta)} .
$$

Then,

$$
\left(\begin{array}{c}
\|x\|_{C_{b}} \\
\|y\|_{C_{b}}
\end{array}\right) \leq(I-M)^{-1}\left(\begin{array}{c}
\sigma_{1} \\
\sigma_{2}
\end{array}\right)
$$

From $(\mathrm{H} 1)$ and $(\mathrm{H} 2)$, we deduce that the solution $(x, y)$ is bounded.

For the next result, we prove the continuous dependence of solutions on initial conditions.

Theorem 16. Assume that (H1) and (H2) hold. If $f(t, 0,0)$ $=g(t, 0,0)=0, t \in J$ and the matrix $M$ defined in (27) converges to zero.

For every $\left(x_{0}, y_{0}\right) \in \mathbb{R} \times \mathbb{R}$, we denoted by $\left(x\left(t, x_{0}\right), y(t\right.$, $\left.\left.y_{0}\right)\right)$ the solution of problem (2). Then, the map $\left(x_{0}, y_{0}\right) \longrightarrow$ $\left(x\left(\cdot, x_{0}\right), y\left(\cdot, y_{0}\right)\right)$ is continuous.

Proof. From Theorem 15, for each initial condition $\left(x_{0}, y_{0}\right)$, $\left(\bar{x}_{0}, \bar{y}_{0}\right) \in \mathbb{R} \times \mathbb{R}$, there exists unique solution $\left(x\left(\cdot, x_{0}\right), y(\cdot\right.$, $\left.\left.y_{0}\right)\right),\left(\bar{x}\left(\cdot, \bar{x}_{0}\right), \bar{y}\left(\cdot, \bar{y}_{0}\right)\right)$, then we get

$$
\begin{aligned}
& \left\{\begin{array}{l}
x\left(t, x_{0}\right)=x_{0}+\frac{1}{\Gamma(\alpha)} \int_{0}^{t}(t-s)^{\alpha-1} f(s, x(s), y(s)) d s, \\
y\left(t, y_{0}\right)=y_{0}+\frac{1}{\Gamma(\beta)} \int_{0}^{t}(t-s)^{\beta-1} g(s, x(s), y(s)) d s,
\end{array}\right. \\
& \left\{\begin{array}{l}
\bar{x}\left(t, \bar{x}_{0}\right)=\bar{x}_{0}+\frac{1}{\Gamma(\alpha)} \int_{0}^{t}(t-s)^{\alpha-1} f(s, \bar{x}(s), \bar{y}(s)) d s, \\
\bar{y}\left(t, \bar{y}_{0}\right)=\bar{y}_{0}+\frac{1}{\Gamma(\beta)} \int_{0}^{t}(t-s)^{\beta-1} g(s, x(s), \bar{y}(s)) d s .
\end{array}\right.
\end{aligned}
$$


Therefore,

$$
\begin{aligned}
\left|x\left(t, x_{0}\right)-\bar{x}\left(t, \bar{x}_{0}\right)\right| \leq & \left|x_{0}-\bar{x}_{0}\right|+\frac{1}{\Gamma(\alpha)} \int_{0}^{t}\left[\left(h_{1, \alpha}(s)|x(s)-\bar{x}(s)|\right.\right. \\
& \left.+h_{2, \alpha}(s)|y(s)-\bar{y}(s)|\right] d s, \\
\left|y\left(t, y_{0}\right)-\bar{y}\left(t, \bar{y}_{0}\right)\right| \leq & \left|y_{0}-\bar{y}_{0}\right|+\frac{1}{\Gamma(\beta)} \int_{0}^{t}\left[\left(h_{3, \beta}(s)|x(s)-\bar{x}(s)|\right.\right. \\
& \left.+h_{4, \beta}(s)|y(s)-\bar{y}(s)|\right] d s .
\end{aligned}
$$

Hence,

$$
\begin{aligned}
\left(\begin{array}{c}
\left\|x\left(\cdot, x_{0}\right)-\bar{x}\left(\cdot, \bar{x}_{0}\right)\right\| C_{b} \\
\left\|y\left(\cdot, y_{0}\right)-\bar{y}\left(\cdot, \bar{y}_{0}\right)\right\| C_{b}
\end{array}\right) & \leq(I-M)^{-1}\left(\begin{array}{c}
\left|x_{0}-\bar{x}_{0}\right| \\
\left|y_{0}-\bar{y}_{0}\right|
\end{array}\right) \\
& \longrightarrow 0, \quad \text { as }\left(x_{0}, y_{0}\right) \longrightarrow\left(\bar{x}_{0}, \bar{y}_{0}\right) .
\end{aligned}
$$

\section{Existence and Compactness of Solution Sets}

For the existence and compactness result of problem (2), we consider the following Banach space:

$$
\mathrm{BC}_{b}(J, \mathbb{R})=\left\{u \in C(J, \mathbb{R}): \sup _{t \in J} \frac{|u(t)|}{1+t}<\infty\right\},
$$

with norm

$$
\|u\|_{B}=\sup _{t \in J} \frac{|u(t)|}{1+t} .
$$

It is evident that $\mathrm{BC}_{b}(J, \mathbb{R})$ is a Banach space. The following compactness criterion on unbounded domains is called Corduneanu compactness criterion in which the proof is easy and similar to the classical one in $C_{b}\left(\mathbb{R}^{+}, \mathbb{R}\right)$ (see [38]).

Lemma 17. Let $H \subset B C_{b}\left(\mathbb{R}^{+}, \mathbb{R}\right)$. Then, $H$ is relatively compact if the following conditions hold:

(a) $H$ is uniformly bounded in $B C_{b}\left(\mathbb{R}^{+}, \mathbb{R}^{n}\right)$

(b) The functions belonging to $H$ are almost equicontinuous on $\mathbb{R}^{+}$, i.e., for all $I \subset \mathbb{R}_{+}$compact interval, for any $\epsilon>0$, there exists $\delta(\epsilon)>0$ such that for every $t_{1}, t_{2} \in J$ with $\left|t_{1}-t_{2}\right|<\delta(\epsilon)$, we have for all $u \in H$

$$
\left|\frac{u\left(t_{2}\right)}{1+t_{2}}-\frac{u\left(t_{1}\right)}{1+t_{1}}\right|<\epsilon
$$

(c) The functions from $H$ are equiconvergent, that is, given $\epsilon>0$, there corresponds $T(\epsilon)>0$ such that

$$
\left|\frac{u\left(t_{1}\right)}{1+t_{1}}-\frac{u\left(t_{2}\right)}{1+t_{2}}\right|<\epsilon \quad \text { for any } t_{1}, t_{2} \geq(\epsilon) \text { and } u \in H
$$

In the sequel of this section, we will consider the following assumption.

(H3). For every $l>0$, the functions $(x, y) \longrightarrow f(t, x, y), g(t$, $x, y)$ are uniformly continuous on the sets $[-l, l] \times[-l, l]$ uniformly with respect to $t \in \mathbb{R}_{+}$, i.e., $f$ and $g$ satisfied the following condition: for all $l>0, \epsilon>0$, there exists $\delta(\epsilon)>0$ such that for all $(x, y),(\bar{x}, \bar{y}) \in[-l, l] \times[-l, l]$ and for all $t \in \mathbb{R}_{+}$with $|x /(1+t)-\bar{x} /(1+t)|<\delta(\epsilon),|y /(1+t)-\bar{y} /(1+t)|<\delta(\epsilon)$, we have

$$
|f(t, x, y)-f(t, \bar{x}, \bar{y})|<\epsilon,|g(t, x, y)-g(t, \bar{x}, \bar{y})|<\epsilon .
$$

(H4). There exist $a_{i}, b_{i}, c_{i}>0, i=1,2$ such that

$$
\begin{aligned}
&|f(t, x, y)| \leq a_{1} \frac{|x|}{1+t}+b_{1} \frac{|y|}{1+t}+c_{1}, \\
&|g(t, x, y)| \leq a_{2} \frac{|x|}{1+t}+b_{2} \frac{|y|}{1+t}+c_{2}, \\
& \forall x, y \in \mathbb{R}, t \in \mathbb{R}_{+} .
\end{aligned}
$$

Theorem 18. Assume that (H3) and (H4) hold. Then, the problem (2) has at least one bounded solution. Moreover, the solution set

$$
\begin{aligned}
S\left(x_{0}, y_{0}\right)= & \left\{(x, y) \in B C_{b}(J, \mathbb{R})\right. \\
& \left.\times B C_{b}(J, \mathbb{R}):(x, y) \text { is solution of }(2)\right\},
\end{aligned}
$$

is compact and the multivalued map $S:\left(x_{0}, y_{0}\right) \longrightarrow S\left(x_{0}, y_{0}\right)$ is u.s.c.

Proof. Let $N=\left(N_{1}, N_{2}\right)$ is defined in the proof of Theorem 15.

Step 1. $N$ is well defined. Let $(x, y) \in \mathrm{BC}_{b}(J, \mathbb{R}) \times \mathrm{BC}_{b}(J, \mathbb{R})$, then

$$
\begin{aligned}
\frac{\left|N_{1}(x(t), y(t))\right|}{1+l} \leq & \frac{\left|x_{0}\right|}{1+l}+\frac{1}{(1+l) \Gamma(\alpha)} \int_{0}^{t} \\
& \cdot(t-s)^{\alpha-1}|f(s, x(s), y(s))| d s \\
\leq & \frac{\left|x_{0}\right|}{1+l} \frac{1}{(1+l) \Gamma(\alpha)} \int_{0}^{t}(t-s)^{\alpha-1} \\
& \cdot\left(a_{1} \frac{(|x(s)|)}{1+s}+b_{1} \frac{(|y(s)|)}{1+s}+c_{1}\right) d s \\
\leq & \frac{\left|x_{0}\right|}{1+l}+\frac{c_{1} t^{\alpha}}{\Gamma(\alpha+1)(1+t)} \\
& +\frac{1}{(1+t) \Gamma(\alpha)} \int_{0}^{t}(t-s)^{\alpha-1} \\
& \cdot\left(a_{1} \frac{(|x(s)|)}{1+s}+b_{1} \frac{(|y(s)|)}{1+s}+\right) d s \\
\leq & \left|x_{0}\right|+\frac{c_{1}}{\Gamma(\alpha+1)}+\frac{1}{(1+t) \Gamma(\alpha)} \int_{0}^{t} \\
& \cdot(t-s)^{\alpha-1} d s\left(a_{1}\|x\|_{B}+b_{1}\|y\|_{B}\right) .
\end{aligned}
$$


Hence,

$$
\begin{aligned}
& \sup _{t \in J} \frac{\left|N_{1}(x(t), y(t))\right|}{1+t} \leq\left|x_{0}\right|+\frac{c_{1}}{\Gamma(\alpha+1)}+\frac{a_{1}\|x\|_{B}+b_{1}\|y\|_{B}}{\Gamma(\alpha+1)}, \\
& \sup _{t \in J} \frac{\left|N_{2}(x(t), y(t))\right|}{1+t} \leq\left|y_{0}\right|+\frac{c_{1}}{\Gamma(\beta+1)}+\frac{a_{2}\|x\|_{B}+b_{2}\|y\|_{B}}{\Gamma(\beta+1)} .
\end{aligned}
$$

Step 2. $N$ is continuous.

Let $\left(x_{n}, y_{n}\right) \longrightarrow(x, y)$ in $\mathrm{BC}_{b}(J, \mathbb{R}) \times \mathrm{BC}_{b}(J, \mathbb{R})$. Then, there exists $l>0$ such that for any $t \in \mathbb{R}_{+}$and $n \in \mathbb{N}$, we have

$$
\frac{x_{n}(t)}{1+t}, \frac{y_{n}(t)}{1+t}, \frac{x(t)}{1+t}, \frac{y(t)}{1+t} \in[-l, l] .
$$

By (H3), for every $\epsilon>0$, there exists $\delta(\epsilon)>0$ such that for all $x, y, \bar{x}, \bar{y} \in[-l, l]$ and for all $t \in \mathbb{R}_{+}$with $\mid x /(1+t)-\bar{x} /(1$ $+t)|<\delta(\epsilon)| y /,(1+t)-\bar{y} /(1+t) \mid<\delta(\epsilon)$ we have

$$
\begin{aligned}
& |f(t, x, y)-f(t, \bar{x}, \bar{y})| \\
& \quad<\epsilon \Gamma(\alpha+1),|g(t, x, y)-g(t, \bar{x}, \bar{y})|<\epsilon \Gamma(\beta+1) .
\end{aligned}
$$

Since $\left(x_{n}, y_{n}\right)$ converge to $(x, y)$, then there exists $n_{0} \in \mathbb{N}$ such that for all $n>n_{0}$,

$$
\left|\frac{x_{n}(t)}{1+t}-\frac{x(t)}{1+t}\right|<\delta(\epsilon),\left|\frac{y_{n}(t)}{1+t}-\frac{y(t)}{1+t}\right|<\delta(\epsilon), \quad \forall t \in \mathbb{R}_{+} .
$$

Hence,

$$
\begin{aligned}
& \left|\frac{N_{1}\left(x_{n}, y_{n}\right)(t)}{1+t}-\frac{N_{1}(x, y)(t)}{1+t}\right| \\
& \quad \leq \frac{1}{\Gamma(\alpha)} \int_{0}^{t} \frac{(t-s)^{\alpha-1}}{1+t}\left|f\left(s, x_{n}(s), y_{n}(s)\right)-f(s, x(s), y(s))\right| d s \\
& \quad \leq \frac{1}{\Gamma(\alpha)} \int_{0}^{t} \frac{(t-s)^{\alpha-1}}{1+t} d s \Gamma(\alpha+1) \epsilon \leq \frac{t^{\alpha}}{1+t} \epsilon \leq \epsilon .
\end{aligned}
$$

Thus

$$
\begin{aligned}
\left\|N_{1}\left(x_{n}, y_{n}\right)-N_{1}(x, y)\right\|_{B} & \longrightarrow 0, \\
\left\|N_{2}\left(x_{n}, y_{n}\right)-N_{2}(x, y)\right\|_{B} & \longrightarrow 0, \\
\text { as } n & \longrightarrow \infty .
\end{aligned}
$$

Step 3. We will show that $N$ maps bounded sets into bounded sets in $B C_{b}(J, R) \times B C_{b}(J, R)$.
Let $B_{r}:=\left\{(x, y) \in \mathrm{BC}_{b}(J, \mathbb{R}) \times \mathrm{BC}_{b}(J, \mathbb{R}):\|(x, y)\|_{\infty} \leq r\right\}$, where $r=\left(r_{1}, r_{2}\right)$ and if $(x, y) \in B_{r}$, then we obtain

$$
\begin{aligned}
\left|\frac{N_{1}(x(t), y(t))}{1+t}\right| & \leq \frac{1}{\Gamma(\alpha)} \int_{0}^{t} \frac{(t-s)^{\alpha-1}}{1+t}|f(s, x(s), y(s))| d s \\
& \leq \frac{1}{\Gamma(\alpha)}\left[a_{1} r_{1}+b_{1} r_{2}+c_{1}\right] .
\end{aligned}
$$

Similarly, we have

$$
\left|\frac{N_{2}(x(t), y(t))}{1+t}\right| \leq \frac{1}{\Gamma(\beta)}\left[a_{2} r_{1}+r_{2} b_{2}+c_{2}\right]
$$

Hence,

$$
\left(\left\|N_{1}(x, y)\right\|,\left\|N_{2}(x, y)\right\|\right) \leq\left(l_{1}, l_{2}\right),
$$

where

$$
l_{1}=\frac{1}{\Gamma(\alpha)}\left[a_{1} r_{1}+b_{1} r_{2}+c_{1}\right], l_{2}=\frac{1}{\Gamma(\beta)}\left[a_{2} r_{1}+r_{2} b_{2}+c_{2}\right] .
$$

Step 4. Now, we prove that $N$ maps bounded sets in $\mathrm{BC}_{b}$ $(J, \mathbb{R}) \times \mathrm{BC}_{b}(J, \mathbb{R})$ into almost equicontinuous sets of $\mathrm{BC}_{b}(J, \mathbb{R}) \times \mathrm{BC}_{b}(J, \mathbb{R})$.

Let $(x, y) \in B_{r}$. Then, for all $t_{1}<t_{2}$,

$$
\begin{aligned}
\left|\frac{N_{1}\left(x\left(t_{2}\right), y\left(t_{2}\right)\right)}{1+t_{2}}-\frac{N_{1}\left(x\left(t_{1}\right), y\left(t_{1}\right)\right)}{1+t_{1}}\right| & \\
\leq & \frac{1}{\Gamma(\alpha)} \int_{0}^{t_{1}}\left|\frac{\left(t_{2}-s\right)^{\alpha-1}}{1+t_{2}}-\frac{\left(t_{1}-s\right)^{\alpha-1}}{1+t_{1}}\right| f(s, x(s), y(s)) \mid d s \\
& +\frac{1}{\Gamma(\alpha)} \int_{t_{1}}^{t_{2}} \frac{\left(t_{2}-s\right)^{\alpha-1}}{1+t_{2}}|f(s, x(s), y(s))| d s \\
\leq & \frac{1}{\Gamma(\alpha)} \int_{0}^{t_{1}}\left|\frac{\left(t_{2}-s\right)^{\alpha-1}}{1+t_{2}}-\frac{\left(t_{1}-s\right)^{\alpha-1}}{1+t_{1}}\right|\left(a_{1} r_{1}+b_{1} r_{2}+c_{1}\right) d s \\
& +\frac{1}{\Gamma(\alpha)} \int_{t_{1}}^{t_{2}} \frac{\left(t_{2}-s\right)^{\alpha-1}}{1+t_{2}}\left(a_{1} r_{1}+b_{1} r_{2}+c_{1}\right) d s \\
\leq & \frac{a_{1} r_{1}+b_{1} r_{2}+c_{1}}{\Gamma(\alpha+1)}\left(\left|\frac{t_{2}^{\alpha}}{1+t_{2}}-\frac{t_{1}^{\alpha}}{1+t_{1}}\right|+\frac{2\left(t_{2}-t_{1}\right)^{\alpha}}{1+t_{2}}\right) .
\end{aligned}
$$

Thus,

$$
\left|\frac{N_{1}\left(x\left(t_{2}\right), t\left(t_{2}\right)\right)}{1+t_{2}}-\frac{N_{1}\left(x\left(t_{1}\right), t\left(t_{1}\right)\right)}{1+t_{1}}\right| \longrightarrow 0 \quad \text { as } t_{1} \longrightarrow t_{2} \text {. }
$$


Similarly, we have

$$
\left|\frac{N_{2}\left(x\left(t_{2}\right), y\left(t_{2}\right)\right)}{1+t_{2}}-\frac{N_{2}\left(x\left(t_{1}\right), y\left(t_{1}\right)\right)}{1+t_{1}}\right| \longrightarrow 0 \quad \text { as } t_{1} \longrightarrow t_{2} \text {. }
$$

Step 5. The set $N(\bar{B}(0, r))$ is equiconvergent, i.e., for every $\epsilon>0$, there exists $T(\epsilon)>0$ such that $\mid N\left(x\left(t_{2}\right), y\left(t_{2}\right)\right)-N$ $\left(x\left(t_{1}\right), y\left(t_{1}\right)\right) \mid<\epsilon$, for every $t_{1}, t_{2}>T(\epsilon)$ and each $(x, y) \epsilon$ $\bar{B}(0, r)$.

$$
\begin{aligned}
& \left|\frac{N_{1}\left(x\left(t_{2}\right), y\left(t_{2}\right)\right)}{1+t_{2}}-\frac{N_{1}\left(x\left(t_{1}\right), y\left(t_{1}\right)\right)}{1+t_{1}}\right| \\
& \leq \frac{a_{1} r_{1}+b_{1} r_{2}+c_{1}}{\Gamma(\alpha+1)}\left(\frac{3 t_{2}^{\alpha}}{1+t_{2}}+\frac{t_{1}^{\alpha}}{1+t_{1}}\right), \\
& \left|\frac{N_{2}\left(x\left(t_{2}\right), y\left(t_{2}\right)\right)}{1+t_{2}}-\frac{N_{2}\left(x\left(t_{1}\right), y\left(t_{1}\right)\right)}{1+t_{1}}\right| \\
& \leq \frac{a_{2} r_{1}+b_{1} r_{2}+c_{1}}{\Gamma(\alpha+1)}\left(\frac{3 t_{2}^{\beta}}{1+t_{2}}+\frac{t_{1}^{\beta}}{1+t_{1}}\right) .
\end{aligned}
$$

It is clear that

$$
\frac{3 t_{2}^{\beta}}{1+t_{2}} \longrightarrow 0, \frac{t_{1}^{\beta}}{1+t_{1}} \longrightarrow 0, \quad \text { as } t_{2}, t_{1} \longrightarrow \infty
$$

Then, for any $\epsilon>0$, there exists $T(\epsilon)>0$ such that for all $t_{1}, t_{2}>T(\epsilon)$, we have

$$
\begin{gathered}
\mid \begin{array}{c}
\left|\frac{N_{1}\left(x\left(t_{2}\right), y\left(t_{2}\right)\right)}{1+t_{2}}-\frac{N_{1}\left(x\left(t_{1}\right), y\left(t_{1}\right)\right)}{1+t_{1}}\right| \\
\leq \epsilon, \quad \text { for all }(x, y) \in \bar{B}(0, r),
\end{array} \\
\left|\begin{array}{c}
\frac{N_{2}\left(x\left(t_{2}\right), y\left(t_{2}\right)\right)}{1+t_{2}}-\frac{N_{2}\left(x\left(t_{1}\right), y\left(t_{1}\right)\right)}{1+t_{1}}
\end{array}\right| \\
\leq \epsilon, \quad \text { for any }(x, y) \in \bar{B}(0, r) .
\end{gathered}
$$

Step 6. The set is defined as follows:

$\mathscr{M}=\left\{(x, y) \in \mathrm{BC}_{b}(J, \mathbb{R}) \times \mathrm{BC}_{b}(J, \mathbb{R}):(x, y)=\mu N(x, y)\right.$, $\mu \in(0,1)\}$ is bounded. Let $(x, y) \in \mathscr{M}$, then

$$
\begin{aligned}
\frac{|x(t)|}{1+t} \leq & \frac{\left|x_{0}\right|}{1+t}+\frac{\mu}{(1+t) \Gamma(\alpha)} \int_{0}^{t}(t-s)^{\alpha-1}|f(s, x(s), y(s))| d s \\
\leq & \frac{\left|x_{0}\right|}{1+t}+\frac{\mu}{(1+t) \Gamma(\alpha)} \int_{0}^{t}(t-s)^{\alpha-1} \\
& \cdot\left(a_{1} \frac{|x(s)|}{1+s}+b_{1} \frac{|y(s)|}{1+s}+c_{1}\right) d s
\end{aligned}
$$

$$
\begin{aligned}
\leq & \frac{\left|x_{0}\right|}{1+t}+\frac{c_{1} t^{\alpha}}{\Gamma(\alpha+1)(1+t)} \\
& +\frac{1}{(1+t) \Gamma(\alpha)} \int_{0}^{t}(t-s)^{\alpha-1} \\
& \cdot\left(a_{1} \frac{|x(s)|}{1+s}+b_{1} \frac{|y(s)|}{1+s}\right) d s .
\end{aligned}
$$

Similarly, we get

$$
\begin{aligned}
& \frac{|y(t)|}{1+t}+\frac{\left|y_{0}\right|}{1+t}+\frac{c_{2} t^{\beta}}{\Gamma(\beta+1)(1+t)} \\
& \quad+\frac{1}{(1+t) \Gamma(\beta)} \int_{0}^{t}(t-s)^{\beta-1}\left(a_{2} \frac{|x(s)|}{1+s}+b_{2} \frac{|y(s)|}{1+s}\right) d s .
\end{aligned}
$$

Thus,

$$
\begin{aligned}
\frac{|x(t)|}{1+t}+\frac{|y(t)|}{1+t} \leq & \frac{X_{0}}{1+t}+\frac{C t^{\gamma}}{1+t}+\frac{C_{*}}{1+t} \int_{0}^{t}(t-s)^{\gamma-1} \\
& \cdot\left(\frac{|x(s)|}{1+s}+b_{2} \frac{|y(s)|}{1+s}\right) d s,
\end{aligned}
$$

where

$$
\begin{gathered}
X_{0}=\left|x_{0}\right|+\left|y_{0}\right|, \\
C=\frac{c_{1}}{\Gamma(\alpha+1)}+\frac{c_{2}}{\Gamma(\beta+1)}, \\
C_{*}=\frac{a_{1}+b_{1}}{\Gamma(\alpha)}+\frac{a_{2}+b_{2}}{\Gamma(\beta)}, \\
\gamma=\max (\alpha, \beta) .
\end{gathered}
$$

Hence,

$$
\begin{aligned}
\frac{|x(t)|}{1+t}+\frac{|y(t)|}{1+t} \leq & X_{0}+C+\frac{C_{*}}{1+t} \int_{0}^{t}(t-s)^{\gamma-1} \\
& \cdot\left(\frac{|x(t)|}{1+s}+\frac{|y(s)|}{1+s}\right) d s .
\end{aligned}
$$

By the Gronwall-Bellman Lemma 12, we have

$$
\begin{aligned}
\frac{|x(t)|}{1+t}+\frac{|y(t)|}{1+t} & \leq X_{0}+C+\int_{0}^{t} \sum_{n=1}^{\infty} \frac{\left(C_{*} \Gamma(\gamma)\right)^{n}}{\Gamma(n \gamma)}(t-s)^{n \gamma-1} d s \\
& \leq X_{0}+C+\sum_{n=1}^{\infty} \frac{\left(t^{\gamma} C_{*} \Gamma(\gamma)\right)^{n}}{(1+t)^{n} \Gamma(n \gamma+1)}\left(X_{0}+C\right) \\
& \leq X_{0}+C+\sum_{n=0}^{\infty} \frac{\left(C_{*} \Gamma(\gamma)\right)^{n}}{\Gamma(n \gamma+1)}\left(X_{0}+C\right) .
\end{aligned}
$$


Then, for every $t \in[0, \infty)$, we have

$$
\frac{|x(t)|}{1+t}+\frac{|y(t)|}{1+t} \leq\left(X_{0}+C\right)\left(1+E \gamma\left(C_{*} \Gamma(\gamma)\right):=M,\right.
$$

where $E_{\gamma}$ is the Mittag-Leffler function defined by

$$
E_{\gamma}(z)=\sum_{n=0}^{\infty} \frac{z^{n}}{\Gamma(n \gamma+1)} .
$$

Then,

$$
\|x\|_{C_{b}} \leq M,\|y\|_{C_{b}} \leq M
$$

According to Theorem 7, problem (2) has at least one solution.

Now, we show that the set

$$
\begin{aligned}
S\left(x_{0}, y_{0}\right)= & \left\{(x, y) \in \mathrm{BC}_{b}(J, \mathbb{R})\right. \\
& \left.\times \mathrm{BC}_{b}(J, \mathbb{R}):(x, y) \text { is solution of }(2)\right\},
\end{aligned}
$$

is compact. It is clear that $S\left(x_{0}, y_{0}\right) \subset N\left(S\left(x_{0}, y_{0}\right)\right)$. From (75), we deduced that $S\left(x_{0}, y_{0}\right)$ is bounded sets in $B C_{b}(J, \mathbb{R})$. Since $N$ is compact, then $S\left(x_{0}, y_{0}\right)$ is compact if and only if $S\left(x_{0}, y_{0}\right)$ is closed. Let $\left\{\left(x_{n}, y_{n}\right)\right\}_{n \geq 1} \subset S\left(x_{0}, y_{0}\right)$ be a sequence converge to $(x, y)$. Thus,

$$
\begin{gathered}
x_{n}(t)=x_{0}+\frac{1}{\Gamma(\alpha)}(t-s)^{\alpha-1} f\left(s, x_{n}(s), y_{n}(s)\right) d s, \\
y_{n}(t)=y_{0}+\frac{1}{\Gamma(\beta)}(t-s)^{\beta-1} g\left(s, x_{n}(s), y_{n}(s)\right) d s, \\
t \in J .
\end{gathered}
$$

Similarly to Step 2, we can prove that

$$
\begin{gathered}
x(t)=x_{0}+\frac{1}{\Gamma(\alpha)} \int_{0}^{t}(t-s)^{\alpha-1} f(s, x(s), y(s)) d s, \\
y(t)=y_{0}+\frac{1}{\Gamma(\beta)} \int_{0}^{t}(t-s)^{\beta-1} g(s, x(s), y(s)) d s, \\
t \in J .
\end{gathered}
$$

This implies that $S\left(x_{0}, y_{0}\right)$ is compact.

The solution operator $S$ is u.s.c.

(a) $S$ has a closed graph:

To see this, first note that the graph of $S$ is the set

$$
\begin{aligned}
G_{S}=\{ & ((a, b),(x, y)) \in(\mathbb{R} \times \mathbb{R}) \\
& \left.\times\left(\mathrm{BC}_{b}(J, \mathbb{R}) \times \mathrm{BC}_{b}(J, \mathbb{R})\right) \mid(x, y) \in S(a, b)\right\} .
\end{aligned}
$$

Let $\left(\left(a_{q}, b_{q}\right),\left(x_{q}, y_{q}\right)\right)_{q}$ be a sequence in $G_{S}$, and let $\left(\left(a_{q}, b_{q}\right),\left(x_{q}, y_{q}\right)\right)_{q} \longrightarrow((a, b),(x, y))$ as $q \longrightarrow \infty$.
Since $\left(x_{q}, y_{q}\right) \in S\left(a_{q}, b_{q}\right)$, then we have

$$
\begin{gathered}
x_{q}(t)=a_{q}+\frac{1}{\Gamma(\alpha)} \int_{0}^{t}(t-s)^{\alpha-1} f\left(s, x_{q}(s), y_{q}(s)\right) d s, \\
y_{q}(t)=b_{q}+\frac{1}{\Gamma(\beta)} \int_{0}^{t}(t-s)^{\beta-1} g\left(s, x_{q}(s), y_{q}(s)\right) d s, \\
t \in J .
\end{gathered}
$$

Let

$$
\begin{aligned}
Z(t)= & \left(Z_{1}(t), Z_{2}(t)\right)=\left(a+\frac{1}{\Gamma(\alpha)} \int_{0}^{t}\right. \\
& \cdot(t-s)^{\alpha-1} f(s, x(s), y(s)) d s, b \\
& \left.+\frac{1}{\Gamma(\beta)} \int_{0}^{t}(t-s)^{\beta-1} g(s, x(s), y(s)) d s\right) \quad t \in J .
\end{aligned}
$$

As in Step 2, we can prove that

$$
\left(x_{q}, y_{q}\right) \longrightarrow(x, y) \quad \text { as } q \longrightarrow \infty .
$$

(b) Using the same method as Steps 3 to 4 , we find that for each bounded set $C \subset \mathrm{BC}_{b}(J, \mathbb{R}) \times \mathrm{BC}_{b}(J, \mathbb{R})$, we can show that $S(C)$ is compact.

From (a) and (b), we concluded that, $S$ is u.s.c.

\section{Applications}

In this section, we show the applicability of our main result. We begin by illustrating by Theorem 15 .

Example 19. Consider the problem:

$$
\left\{\begin{array}{l}
{ }^{c} D^{1 / 2} x=h_{1}(t)\left(1+k_{1} \sin |x|+k_{2} \cos |x|\right), \\
{ }^{c} D^{1 / 2} y=h_{2}(t)\left(1+k_{3}|x|+k_{4}|y|\right)^{1 / 2}, \quad t \in \mathbb{R}, \\
x(0)=x_{0}, \\
y(0)=y_{0},
\end{array}\right.
$$

where

$$
\begin{aligned}
h_{1}(t) & =\frac{\sqrt{y} \int_{0}^{t} s e^{-s 2} d s}{100\left(1+t^{2}\right)} \text { and } h_{2}(s) \\
& =\frac{t \sqrt{t}}{200\left(1+t^{2}\right)}, \quad t \in \mathbb{R}_{+}, k_{i} \geq 0, i=1,2,3,4, \\
f(t, x, y) & =h_{1}(t)\left(1+k_{1} \sin |x|+k_{2} \cos |y|\right), g(t, x, y) \\
& =h_{2}(t)\left(1+k_{3}|x|+k_{4}|y|\right) .
\end{aligned}
$$


It is clear that

$$
\begin{aligned}
\int_{0}^{t} \frac{h_{1}(s)}{(t-s)^{1 / 2}} d s & =\int_{0}^{t} \frac{\sqrt{s} \int_{0}^{s} r e^{-r^{2}} d r}{100\left(1+s^{2}\right)(t-s)^{1 / 2}} \\
& \leq \frac{\int_{0}^{\infty} e^{-r^{2}} d r}{100\left(1+s^{2}\right)(t-s)^{1 / 2}} \int_{0}^{t} \frac{s \sqrt{s}}{\left(1+s^{2}\right)(t-s)^{1 / 2}} d s \\
& =\frac{\int_{0}^{\infty} e^{-r^{2}} d r}{100} \int_{0}^{t} \frac{t^{2} l \sqrt{l}}{\left(1+(t l)^{2}\right)(1-l)^{1 / 2}} d l \\
& \leq \frac{\int_{0}^{\infty} e^{-r^{2}} d r}{100} \int_{0}^{t}(1-l)^{-(1 / 2)} d l \\
& \leq \frac{\int_{0}^{\infty} e^{-r^{2}} d r}{50}=\frac{\sqrt{\pi}}{100}, \\
\int_{0}^{t} \frac{h_{2}(s)}{(t-s)^{1 / 2}} d s & =\int_{0}^{t} \frac{s \sqrt{s}}{200\left(1+s^{2}\right)(t-s)^{1 / 2}} d s=\leq \frac{1}{100}
\end{aligned}
$$

Then,

$$
\sup _{t \in \mathbb{R}_{+}} \int_{0}^{t} \frac{h_{i}(s)}{(t-s)^{1 / 2}} d s<\infty, \quad i=1,2 .
$$

Observe that every $x, y, \bar{x}, \bar{y} \in \mathbb{R}$ and $t \in \mathbb{R}_{+}$, we have

$$
\begin{aligned}
& |f(t, x, y)-f(t, \bar{x}, \bar{y})| \leq k_{1} h_{1}(t)|x-\bar{x}|+k_{2} h_{1}(t)|y-\bar{y}|, \\
& |g(t, x, y)-g(t, \bar{x}, \bar{y})| \leq k_{3} h_{2}(t)|x-\bar{x}|+k_{4} h_{2}(t)|y-\bar{y}| .
\end{aligned}
$$

Hence, the condition (H1) holds.

We see that

$$
|f(t, 0,0)|=h_{1}(t),|g(t, 0,0)|=h_{2}(t), \quad t \in \mathbb{R}_{+} .
$$

Hence, the condition (H2) holds. Assume that $k_{1} k_{4}-k_{2}$ $k_{3} \neq 0$. For this, we have

$$
\tilde{M}=\left(\begin{array}{cc}
\frac{k_{1} \sqrt{\pi}}{\Gamma(1 / 2) 100} & \frac{k_{2} \sqrt{\pi}}{\Gamma(1 / 2) 100} \\
\frac{k_{3} \sqrt{\pi}}{\Gamma(1 / 2) 100} & \frac{k_{4} \sqrt{\pi}}{\Gamma(1 / 2) 100}
\end{array}\right) .
$$

det $\tilde{M} \neq 0$. If we add that $k_{2}=0$ or $k_{3}$ and $0<k_{1}<(\Gamma(1 /$ 2) 100$) / \sqrt{\pi}, 0<k_{4}<\Gamma(1 / 2) 100 \leq 1$. The $\rho(\bar{M})<1$. By Theorem 15 , it follows that problem (83) has a unique solution.

In this last example, we illustrate the applicability of Theorem 18.

Lemma 20. Let us consider the function $f: \mathbb{R}_{+} \times \mathbb{R} \times \mathbb{R}$ $\longrightarrow \mathbb{R}$ defined by

$$
f(t, x, y)=a(t) \phi(x, y)
$$

where $a: \mathbb{R}_{+} \longrightarrow \mathbb{R}, \phi: \mathbb{R} \times \mathbb{R} \longrightarrow \mathbb{R}$ satisfy the following assumptions:

(S1) $a \in C_{b}(\mathbb{R})$.

(S2) The function $\varphi$ is continuous and bounded. Then, the function

$$
f_{*}(t, x, y)=a(t) \phi\left(\frac{x}{1+t}, \frac{y}{1+t}\right), \quad t \in \mathbb{R}_{+},(x, y) \in \mathbb{R} \times \mathbb{R},
$$

satisfied the condition (H3).

Proof. Since $a$ is bounded, then there exists $A>0$ such that

$$
|a(t)|<A, \quad \forall t \in \mathbb{R}_{+} .
$$

Let $l>0$, then for any $(x, y),(\bar{x}, \bar{y}) \in[-l, l] \times[-l, l]$, we obtain

$$
|f(t, x, y)-f(t, \bar{x}, \bar{y})|=|a(t)||\phi(x, y)-\phi(\bar{x}, \bar{y})| .
$$

Hence,

$$
|f(t, x, y)-f(t, \bar{x}, \bar{y})| \leq A|\phi(x, y)-\phi(\bar{x}, \bar{y})| .
$$

It is clear that form (S2) $\phi$ is uniformly continuous on the $[-l, l] \times[-l, l]$. Thus, for any $\epsilon>0$ there exists $\delta(\epsilon)>0$ such that for all $(x, y),(\bar{x}, \bar{y}) \in[-l, l] \times[-l, l]$ with $|x-\bar{x}|<\delta(\epsilon), \mid y$ $-\bar{y} \mid<\delta(\epsilon)$ then,

$$
|f(t, x, y)-f(t, \bar{x}, \bar{y})| \leq A \epsilon .
$$

This implies that for every $t \in \mathbb{R}_{+}$such that for all $(x, y)$, $(\bar{x}, \bar{y}) \in[-l, l] \times[-l, l]$ with $|x /(1+t)-\bar{x} /(1+t)|<\delta(\epsilon), \quad \mid y /$ $(1+t)-\bar{y} /(1+t) \mid<\delta(\epsilon)$, we get

$$
|f(t, x, y)-f(t, \bar{x}, \bar{y})| \leq A \epsilon .
$$

Theorem 21. Let $a_{1}, a_{2}: \mathbb{R}_{+} \longrightarrow \mathbb{R}, \phi_{1}, \phi_{2}: \mathbb{R} \times \mathbb{R} \longrightarrow \mathbb{R}$ be functions that satisfy the conditions (S1) and (S2).

Assume

(S3) There exists $d_{i}>0, i=1,4$ such that

$$
\begin{array}{ll}
\left|\phi_{1}(x, y)\right| \leq d_{1}|x|+d_{2}|y|+d_{3}, & \forall x, y \in \mathbb{R}, t \in \mathbb{R}_{+}, \\
\left|\phi_{2}(x, y)\right| \leq d_{4}|x|+d_{5}|y|+d_{6}, & \forall x, y \in \mathbb{R}, t \in \mathbb{R}_{+} .
\end{array}
$$

Then, the following problem

$$
\left\{\begin{array}{l}
{ }^{c} D^{\alpha} x(t)=f(t, x,(t)), \\
{ }^{c} D^{\beta} x(t)=g(t, x,(t)), \quad t \in J, \\
x(0)=x_{0}, \\
y(0)=y_{0},
\end{array}\right.
$$


where

$$
\begin{array}{ll}
f(t, x, y)=a_{1}(t) \phi_{1}\left(\frac{x}{1+t}, \frac{y}{1+t}\right), & t \in \mathbb{R}_{+}, x, y \in \mathbb{R}, \\
g(t, x, y)=a_{2}(t) \phi_{2}\left(\frac{x}{1+t}, \frac{y}{1+t}\right), & t \in \mathbb{R}_{+}, x, y \in \mathbb{R} .
\end{array}
$$

Proof. From Lemma 20, we deduce that $f$ and $g$ satisfies (H3). By (S1) and (S3), there exist $A_{1}, A_{2}>0$ such that

$$
\begin{aligned}
& f(t, x, y) \leq A_{1}\left(\frac{|x|}{1+t}, \frac{|y|}{1+t}\right) \\
& g(t, x, y) \leq A_{2}\left(\frac{x}{1+t}, \frac{y}{1+t}\right), \quad t \in \mathbb{R}_{+}, x, y \in \mathbb{R} .
\end{aligned}
$$

Therefore, all the conditions of Theorem 18 hold. Then, problem (98) has at least one solution in $\mathrm{BC}_{b}$.

Remark 22. For another application, we can replace the condition (S2) with (S4). The functions $\varphi_{1}$ and $\varphi_{2}$ are uniformly continuous and bounded.

\section{Conclusion}

In this paper, we investigated a system of fractional differential equations under various assumptions on the righthand-side nonlinearity and we obtain a number of results regarding the existence and uniqueness of solutions in an appropriate space of continuous functions. In this paper, we have focused on the dependence continuity of a solution, compactness of solution sets, and upper semicontinuity of operator solutions. We hope this paper can provide some contribution to the questions of existence and topological structure for the system of fractional differential equations on unbounded domains.

\section{Data Availability}

No data were used to support this study.

\section{Conflicts of Interest}

The authors declare no conflicts of interest.

\section{Acknowledgments}

The research of Juan J. Nieto has been partially supported by the Agencia Estatal de Investigacin (AEI) of Spain, cofinanced by the European Fund for Regional Development (FEDER) corresponding to the 2014-2020 multiyear financial framework, project MTM2016-75140-P, and by Xunta de Galicia under grant ED431C 2019/02.

\section{References}

[1] D. Baleanu, Z. B. Güvenç, and J. A. T. Machado, New Trends in Nanotechnology and Fractional Calculus Applications, Springer, New York, 2010.
[2] X. L. Ding and J. J. Nieto, "Analysis and numerical solutions for fractional stochastic evolution equations with almost sectorial operators," Journal of Computational and Nonlinear Dynamics, vol. 14, no. 9, article 091001, 2019.

[3] N. D. Cong, H. T. Tuan, and H. Trinh, "On asymptotic properties of solutions to fractional differential equations," Journal of Mathematical Analysis and Applications, vol. 484, no. 2, article 123759, 2020.

[4] E. F. Doungmo Goufo and J. J. Nieto, "Attractors for fractional differential problems of transition to turbulent flows," Journal of Computational and Applied Mathematics, vol. 339, pp. 329$342,2018$.

[5] A. A. Kilbas, H. M. Srivastava, and J. J. Trujillo, "Theory and Applications of Fractional Differential Equations," in NorthHolland Mathematics Studies, vol. 204, Elsevier Science B.V., Amsterdam, 2006.

[6] F. Mainardi, "Fractional calculus: some basic problems in continuum and statistical mechanics," in Fractals and Fractional Calculus in Continuum Mechanics, A. Carpinteri and F. Mainardi, Eds., pp. 291-348, Springer-Verlag, Wien, 1997.

[7] V. E. Tarasov, Fractional Dynamics: Application of Fractional Calculus to Dynamics of Particles, Fields and Media, Springer, Heidelberg, Higher Education Press, Beijing, 2010.

[8] S. Abbas, M. Benchohra, and G. M. N'Guérékata, Topics in Fractional Diferential Equations, SpringerVerlag, New York, 2012.

[9] B. Ahmad, A. Alsaedi, S. K. Ntouyas, and J. Tariboon, Hadamard-Type Fractional Differential Equations, Inclusions and Inequalities, Springer, 2017.

[10] D. Baleanu, K. Diethelm, E. Scalas, and J. J. Trujillo, Fractional Calculus: Models and Numerical Methods, World Scientific, 2012.

[11] A. B. Malinowska and D. F. M. Torres, Introduction to the Fractional Calculus of Variations, Imperial College Press, London, 2012.

[12] I. Podlubny, Fractional Differential Equations, Academic Press, San Diego, 1999.

[13] Y. Zhou, Basic Theory of Fractional Differential Equations, World Scientific Publishing Co. Pte. Ltd., Hackensack, NJ, 2014.

[14] K. Diethelm, The Analysis of Fractional Differential Equations: an Application-Oriented Exposition Using Differential Operators of Caputo Type, vol. 2010 of Lecture Notes in Mathematics, Springer-Verlag, Berlin, 2004.

[15] M. A. Alqudah, C. Ravichandran, T. Abdeljawad, and N. Valliammal, "New results on Caputo fractional-order neutral differential inclusions without compactness," Advances in Difference Equations, vol. 2019, no. 1, Article ID 528, 2019.

[16] M. Benchohra, J. Henderson, S. K. Ntouyas, and A. Ouahab, "Existence results for fractional order functional differential equations with infinite delay," Journal of Mathematical Analysis and Applications, vol. 338, no. 2, pp. 1340-1350, 2008.

[17] M. Benchohra, J. Henderson, S. K. Ntouyas, and A. Ouahab, "Existence results for fractional functional differential inclusions with infinite delay and application to control theory," Fractional Calculus and Applied Analysis, vol. 11, pp. 35-56, 2008.

[18] C. Ravichandran and D. Baleanu, "Existence results for fractional neutral functional integro-differential evolution equations with infinite delay in Banach spaces," Advances in Difference Equations, vol. 2013, no. 1, Article ID 215, 2013. 
[19] J. Henderson and A. Ouahab, "Impulsive differential inclusions with fractional order," Computers \& Mathematcs with Applications, vol. 59, no. 3, pp. 1191-1226, 2010.

[20] M. Seghier, A. Ouahab, and J. Henderson, "Random solutions to a system of fractional differential equations via the Hadamard fractional derivative," The European Physical Journal Special Topics, vol. 226, no. 16-18, pp. 3525-3549, 2017.

[21] C. Ravichandran, K. Jothimani, H. M. Baskonus, and N. Valliammal, "New results on nondensely characterized integrodifferential equations with fractional order," The European Physical Journal Plus, vol. 133, no. 3, article 109, 2018.

[22] A. I. Perov, "On the Cauchy problem for a system of ordinary differential equations, differential equations," Pviblizhen. Met. Reshen. Differ. Uvavn., vol. 2, pp. 115-134, 1964.

[23] A. I. Perov and A. V. Kibenko, "On a certain general method for investigation of boundary value problems," Izvestiya Rossiiskoi Akademii Nauk. Seriya Matematicheskaya, vol. 30, pp. 249-264, 1966.

[24] M. Dezideriu and R. Precup, "Non-resonance and existence for systems of non-linear operator equations," Applicable Analysis, vol. 87, no. 9, pp. 1005-1018, 2008.

[25] R. Precup, "The role of matrices that are convergent to zero in the study of semilinear operator systems," Mathematical and Computer Modelling, vol. 49, no. 3-4, pp. 703-708, 2009.

[26] H. Berrezoug, J. Henderson, and A. Ouahab, "Existence and uniqueness of solutions for a system of impulsive differential equations on the half-line," Journal of Nonlinear Functional Analysis, vol. 2017, no. 1, article 38, pp. 1-16, 2017.

[27] O. Bolojan-Nica, G. Infante, and R. Precup, "Existence results for systems with coupled nonlocal initial conditions," Nonlinear Analysis: Theory, Methods \& Applications, vol. 94, pp. 231242, 2014.

[28] J. R. Graef, J. Henderson, and A. Ouahab, Topological Methods for Differential Equations and Inclusions, Monographs and Research Notes in Mathematics Series Profile, CRC Press, Boca Raton, FL, 2019.

[29] O. Nica, "Initial-value problems for first-order differential systems with general nonlocal conditions," Electronic Journal of Differential Equations, vol. 2012, no. 74, pp. 1-15, 2012.

[30] R. Precup and A. Viorel, "Existence results for systems of nonlinear evolution equations," International Journal of Pure and Applied Mathematics, vol. 47, pp. 199-206, 2008.

[31] J. D. Murray, Mathematical Biology, Springer, Berlin, 1989.

[32] B. S. Jensen, The Dynamic Systems of Basic Economic Growth Models, Kluwer, Dordrecht, 1994.

[33] R. S. Varga, "Matrix Iterative Analysis," in 2nd Revised and Expanded, Ser, Springer series in Computational Mathematics, Springer, Berlin, 2000.

[34] I. A. Rus, Generalized Contractions and Applications, Cluj University Press, Cluj-Napoca, 2001.

[35] A. Viorel, Contributions to the study of nonlinear evolution equations, nD. thesis, Babe,s-Bolyai University Cluj-Napoca, Department of Mathematics, 2011.

[36] K. Deimling, Multivalued Differential Equations, Walter De Gruyter, Berlin-New York, 1992.

[37] H. Ye, J. Gao, and Y. Ding, "A generalized Gronwall inequality and its application to a fractional differential equation," Journal of Mathematical Analysis and Applications, vol. 328, no. 2, pp. 1075-1081, 2007.

[38] C. Corduneanu, Integral Equations and Stability of Feedback Systems, Academic Press, New York, 1973. 